\title{
Validation of a Clinical Risk-scoring Algorithm for Scrub Typhus Severity in South India
}

\author{
Shivali Gulati ${ }^{1} \odot$, Kiran Chunduru $^{2} \odot$, Mridula Madiyal $^{3} \odot$, Maninder S Setia ${ }^{4}$, Kavitha Saravu $^{5} \odot$
}

\begin{abstract}
Background: A clinical risk-scoring algorithm (CRSA) to forecast the scrub typhus severity was developed from two general hospitals in Thailand where patients were classified into three groups-nonsevere, severe, and fatal. In this study, an attempt was made to validate the risk-scoring algorithm for prognostication of scrub typhus severity in India.

Materials and methods: This prospective study was conducted at a hospital in South India between November 2017 and March 2019 . Patients of scrub typhus were categorized into nonsevere, severe, and fatal according to the CRSA. The patients were also grouped into severe and nonsevere according to the definition of severe scrub typhus which was used as a gold standard. The obtained CRSA score was validated against the classification based on the definition of severe scrub typhus. Receiver operating characteristics (ROC) curve for the scores was plotted and the Youden's index for optimal cutoff was used.

Results: A total of 198 confirmed cases of scrub typhus were included in the study. According to the ROC curve, at a severity score $\geq 7$, an optimal combination of sensitivity of $75.9 \%$ and specificity of $77.5 \%$ was achieved. It correctly predicted $76.77 \%$ (152 of 198 ) of patients as severe, with an underestimation of $10.61 \%$ (21 patients) and an overestimation of $12.63 \%$ ( 25 patients).

Conclusion: In the present study setting, a cutoff of $\geq 7$ for severity prediction provides an optimum combination of sensitivity and specificity. These findings need to be validated in further studies.

Keywords: Clinical Risk-scoring Algorithm, Prognosis, Scrub typhus, Severe scrub typhus.

Indian Journal of Critical Care Medicine (2021): 10.5005/jp-journals-10071-23828
\end{abstract}

\section{INTRODUCTION}

Scrub typhus is one of the rickettsial infections in India caused by the organism Orientia tsutsugamushi. The infection is spread by the bite of larval stage of infected mites or chiggers belonging to the family Trombiculidae. ${ }^{1}$ O. tsutsugamushi is endemic to the "tsutsugamushi triangle" which covers Asia and the Western Pacific region including India. ${ }^{2,3}$ Scrub typhus presents as acute fever with cough, breathlessness, myalgia, headache, nausea and vomiting. ${ }^{1,4}$ An eschar may be present at the site of chigger bite. However, the eschar incidence reported in various studies varies from 7 to $97 \% .^{1,5-8}$ The eschar inducing capacity of different strains of $O$. tsutsugamushi is variable. The detection rate of eschar depends on skin color, anatomical location of the eschar, and type of clothing. Eschar is rare in Southeast Asian patients. ${ }^{9}$ It has also been found that the presence of eschar is associated with severe disease involving respiratory, hematological, and renal system with a higher mortality rate. ${ }^{10}$

The disease is self-limiting but delay in diagnosis and initiation of appropriate treatment may lead to severe complications and mortality up to $30 \% .^{2,4,5,11,12}$ After the first week of illness complications such as jaundice, pneumonia, renal failure, acute respiratory distress syndrome (ARDS), hepatitis, septic shock, myocarditis, and meningoencephalitis may occur in varying proportion of patients. ${ }^{1,5,11-15}$ The low index of clinical suspicion and nonspecific clinical features which overlap with other tropical infections make the diagnosis challenging. ${ }^{16-18}$ Often there is a delay in diagnosis and failure to recognize the severe cases early, leading to complications and mortality.

Thus, prognostication of disease severity may help identify those with severe disease and early initiation of aggressive treatment. Such prognostication scores already exist for many
${ }^{1}$ Department of Medicine, Kasturba Medical College, Manipal Academy of Higher Education, Manipal, Karnataka, India

2,5Department of Infectious Diseases, Kasturba Medical College, Manipal Academy of Higher Education, Manipal, Karnataka, India; Manipal Center for Infectious Diseases, Prasanna School of Public Health, Manipal Academy of Higher Education, Manipal, Karnataka, India

${ }^{3}$ Department of Microbiology, Kasturba Medical College, Manipal Academy of Higher Education, Manipal, Karnataka, India

${ }^{4}$ MGM Institute of Health Sciences, Navi Mumbai, Maharashtra, India

Corresponding Author: Kavitha Saravu, Department of Infectious Diseases, Kasturba Medical College, Manipal Academy of Higher Education, Manipal, Karnataka, India; Manipal Center for Infectious Diseases, Prasanna School of Public Health, Manipal Academy of Higher Education, Manipal, Karnataka, India, Phone: +91 9448107636, e-mail: kavithasaravu@gmail.com

How to cite this article: Gulati S, Chunduru K, Madiyal M, Setia MS, Saravu K. Validation of a Clinical Risk-scoring Algorithm for Scrub Typhus Severity in South India. Indian J Crit Care Med 2021;25(5):551-556.

Source of support: Nil

Conflict of interest: None

other diseases like pneumonia, ${ }^{19}$ dengue fever, ${ }^{20}$ and dengue hemorrhagic fever, ${ }^{21}$ clostridium difficile infection, ${ }^{22}$ and malaria. ${ }^{23}$ Similar scoring system was developed recently in Thailand for scrub typhus. ${ }^{24} \mathrm{~A}$ clinical risk-scoring algorithm (CRSA) was created to forecast the scrub typhus severity among 526 patients diagnosed with scrub typhus from two general hospitals in Thailand during 2004 to 2010. This algorithm was validated using an independent dataset comprising of 257 scrub typhus patients in one of the hospitals. ${ }^{25}$ 
India although one among the endemic regions, does not have an algorithm based on the Indian population for severe scrub typhus. The purpose of this study was to validate the CRSA for the application of regular practice in our country by including data of confirmed cases of scrub typhus. The scoring system if validated may assist routine clinical guidelines to improve patient management in the early stages of the disease.

\section{Materials and Methods}

In this prospective study conducted at a hospital in South India from November 2017 to March 2019, data of 260 consecutive hospitalized cases of scrub typhus were screened. Out of these, patients with age more than 18 years diagnosed with scrub typhus by immunofluorescence or IgM ELISA were included in the study. Patients with other concomitant infections were excluded. Consequently, 198 patients were included in the study. For diagnosis using ELISA, an optical density value of $>0.5$ was taken as positive. For polymerase chain reaction technique, gene targeted was $56 \mathrm{kDa}$ type-specific antigen gene sequence of $O$. tsutsugamushi.

Exposure variables: For all the study subjects, after an informed consent, history, clinical examination, and laboratory parameters at the time of presentation were noted in the proforma. History included demographic details, fever, myalgia, headache, cough, abdominal pain, nausea/vomiting, dyspnea, wheezing, and seizures. The presence of eschar, rash, lymphadenopathy, jaundice was noted. The laboratory values noted for each subject included hemoglobin, total counts, platelets, renal and liver parameters, and urine routine.

Outcome variables: CRSA score (Table 1), and severity as per the standard definition (Table 2). Patient's risk scores were assigned according to the scoring system given in Table 1.

Patients were categorized based on the CRSA score as follows:

i. Nonsevere: CRSA score $\leq 5$

ii. Severe: CRSA score 6-9

iii. Fatal: $C R S A$ score $\geq 10$

The gold standard criteria used for defining the severity of scrub typhus is given in Table 2, as used in the Thailand study. ${ }^{24,25}$ Patients with one or more organ system complications were classified as severe. Patients were treated by the treating clinicians as per their clinical judgment with azithromycin or doxycycline. The final in-hospital outcome of the patient was noted.

\section{Statistical Analysis}

The clinical data of the study cohort were recorded in a predefined proforma and were entered into Microsoft Excel. Stata Version 15. 1 (৫ StataCorp, College Station, Texas, USA) was used for the analysis of the collected data. Patients were stratified based on the risk score developed by Sriwongpan et al. ${ }^{24}$ Simultaneously, the patients were stratified into severe and nonsevere groups according to the gold standard criteria (Table 2). Continuous variables are expressed as mean and standard deviation or median and interquartile range. Categorical variables are expressed as counts and percentages. Differences among the groups are analyzed using the Chi-square test or Fischer's exact test. Severity by CRSA score was validated against the classification based on the gold standard criteria. The

Table 1: Clinical Risk Scoring Algorithm (CRSA) adopted from the Thailand study 24,25

\begin{tabular}{lll}
\hline Predictor & Category & Assigned score \\
\hline Age (years) & $\leq 15$ & 0 \\
& $>15$ & 3 \\
Pulse rate (beats/min) & $\leq 100$ & 0 \\
& $>100$ & 2 \\
Crepitation's & No & 0 \\
& Yes & 2 \\
Aspartate transaminase (IU/L) & $\leq 160$ & 0 \\
& $>160$ & 2 \\
Serum albumin & $\leq 3.0$ & 3 \\
& $>3.0$ & 0 \\
Serum creatinine (mg/dL) & $\leq 1.4$ & 0 \\
Total score & $>1.4$ & 4 \\
\hline
\end{tabular}

Table 2: Definition of organ dysfunctions in severe scrub typhus

\begin{tabular}{|c|c|}
\hline Organ system & Criteria \\
\hline Cardiovascular system & $\begin{array}{l}\text { Presence of any of the following: } \\
\text { - Systolic blood pressure <90 mm Hg } \\
\text { - Abnormal cardiac arrhythmias with no previous history of atrial fibrillation, supraventricular } \\
\text { tachycardia, premature ventricular contraction. } \\
\text { - Myocarditis, elevated creatinine kinase-MB above baseline }\end{array}$ \\
\hline Respiratory system & $\begin{array}{l}\text { Acute respiratory distress syndrome, defined as } \mathrm{PaO}_{2} / \mathrm{FiO}_{2}<200 \mathrm{~mm} \mathrm{Hg} \text {, with bilateral interstitial } \\
\text { infiltration on chest film with normal cardiothoracic ratio, or no volume overload of central venous } \\
\text { pressure from central venous pressure }\end{array}$ \\
\hline Central nervous system & $\begin{array}{l}\text { Presence of any of the following: } \\
\text { - Glasgow Coma Scale } \leq 12 \text { without other causes } \\
\text { - Seizure without other causes, or } \\
\text { - Meningoencephalitis }\end{array}$ \\
\hline Hematology & Platelet count $\leq 20,000 / \mathrm{mm}^{3}$ \\
\hline Urinary tract & Presence of acute renal failure, defined as creatinine $\geq 2 \mathrm{mg} / \mathrm{dL}$ or creatinine change $>0.5 \mathrm{mg} / \mathrm{dL} / \mathrm{day}$ \\
\hline Gastrointestinal/hepatobiliary tract & $\begin{array}{l}\text { Presence of hepatitis, defined as elevated aspartate transaminase or alanine transaminase more than } \\
\text { fivefold }\end{array}$ \\
\hline
\end{tabular}


receiver operating characteristics $(\mathrm{ROC})$ curve for the scores using the Youden's index for optimal cutoff was plotted. A $p$-value of $<0.05$ was considered statistically significant.

\section{Ethics Approval}

The study was approved by the Institutional Ethics Committee with approval number IEC: 636/2017.

\section{Results}

In this prospective study carried out from November 2017 to March 2019, 260 hospitalized patients diagnosed with scrub were screened. All the patients fulfilling the inclusion criteria enrolled in the study were diagnosed by IgM ELISA [198 (100\%)] and immunofluorescence assay [28(14.4\%)]. Among the enrolled, 111 were male $(56.06 \%)$ and 87 were female (43.9\%). Mean age of the cohort was $45.22 \pm 14.29$. The participant enrollment is represented in Flowchart 1.

When the patients were classified according to the CRSA score, 77 patients were predicted to have a nonsevere course, 71 severe, and 50 fatal. According to the gold standard criteria, 111 patients were classified as nonsevere and 87 as severe. Clinical characteristics of the scrub typhus study cohort are given in Table 3. The laboratory parameters of the cohort are described in Table 4. Among 198 patients, three patients died in the hospital. On comparing the duration of stay in the hospital across the three severity groups, patients in the fatal group were found to have a longer duration of the hospital, as well as ICU, stay. Among the 198 patients, 186 (93.9\%) patients recovered, 3 (1.5\%) patients succumbed, and 8 (4\%) patients were discharged against medical advice. These results were statistically significant. The systemic complications of the cohort are described in Table 5.

It was observed that out of 87 patients who were categorized as severe according to the gold standard criteria, 70 had a CRSA score of $\geq 6$. Similarly, out of the 111 patients labeled as nonsevere, 60 patients had a CRSA score $<6$.
Sensitivity and specificity at a cutoff score of $\geq 6$ were found to be 80.5 and $54.1 \%$, respectively. Positive predictive value was found to be $57.9 \%$ and negative predictive value was found to be $77.9 \%$. Positive likelihood ratio calculated was 1.75 and negative likelihood ratio was 0.361 with an odds ratio of 4.84 (Supplementary file).

It was observed that out of the 87 patients who were categorized as severe according to the gold standard criteria, 45 had a CRSA score of $\geq 10$. Similarly, out of the 111 patients labelled as nonsevere, 106 patients had a CRSA score of $<10$.

Sensitivity and specificity at a cutoff point of $\geq 10$ were found to be 51.7 and $95.5 \%$, respectively. Positive predictive value was found to be $90 \%$ and negative predictive value was found to be $71.6 \%$. Positive likelihood ratio calculated was 11.5 and negative likelihood ratio was 0.506 with an odds ratio of 22.7 (Supplementary file).

The ROC curve for the scores was plotted and the Youden's index for optimal cutoff was used (Fig. 1).

The diagnostic CRSA criteria were tested against the existing gold-standard criteria to get an optimal cutoff score.

\section{Discussion}

The present study provides additional evidence on the validity of the CRSA in a setting outside Thailand.

Sriwongpan et al. in their validation of CRSA have identified that in the severe group (score 6-9) $14.4 \%$ (24 out of 55) of patients were correctly classified with an underestimation of $5.4 \%$ (9 patients) and an overestimation of $13.8 \%$ (23 patients). ${ }^{24,25}$ When similar calculations were carried out for the severe group (with a CRSA score of $\geq 6$ ) in our study, a sensitivity of $80.5 \%$ and specificity of $54.1 \%$ were obtained. The severity of $65.66 \%$ (130 of 198) patients was correctly classified, with an underestimation of $8.58 \%$ (17 patients) and an overestimation of $25.75 \%$ (51 patients). Positive predictive value was found to be $57.9 \%$ and negative predictive value was found to be $77.9 \%$.

Flowchart 1: Flowchart of participant enrollment

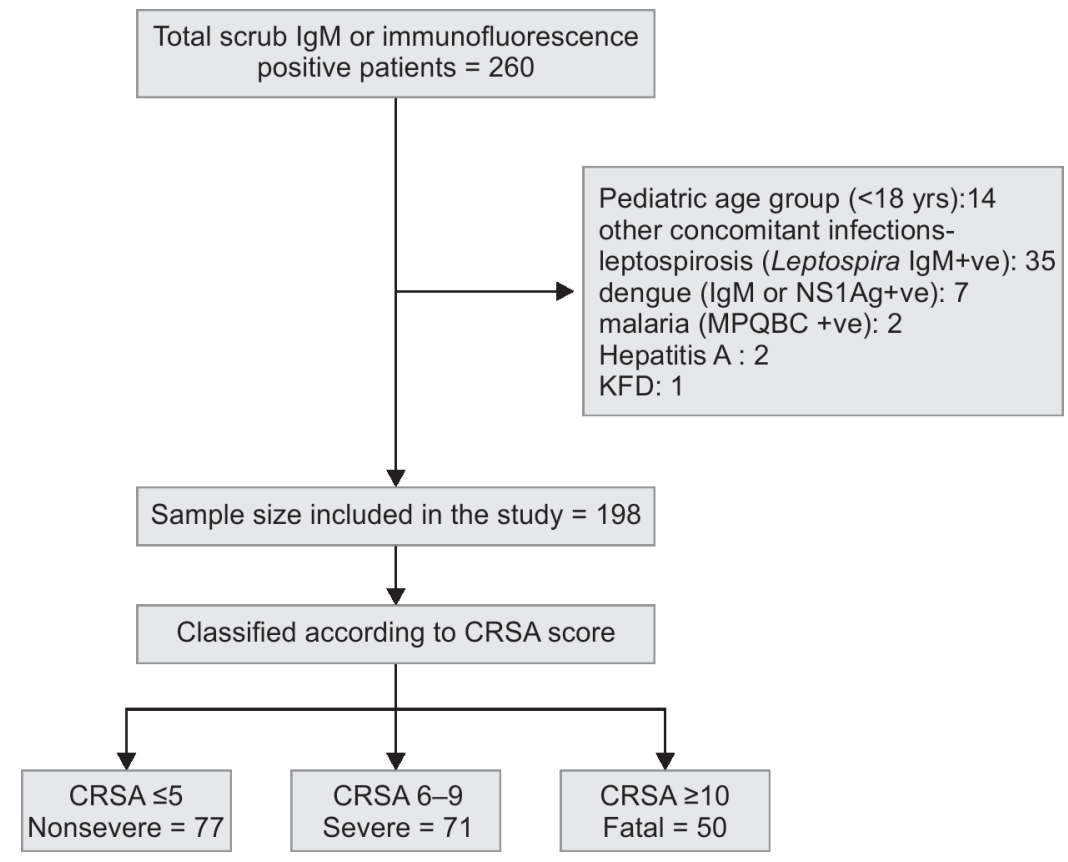


Table 3: Clinical characteristics of the scrub typhus study cohort by severity

\begin{tabular}{|c|c|c|c|c|c|}
\hline Clinical features & $\begin{array}{l}\text { Nonsevere }(C R S A \leq 5) \\
(N=77) n(\%) \text { or } \\
\text { mean }( \pm S D)\end{array}$ & $\begin{array}{l}\text { Severe }(C R S A=6-9) \\
(N=71) n(\%) \text { or } \\
\text { mean }( \pm S D)\end{array}$ & $\begin{array}{l}\text { Fatal }(C R S A \geq 10) \\
(N=50) n(\%) \text { or } \\
\text { mean }( \pm S D)\end{array}$ & $\begin{array}{l}\text { Total n (\%) } \\
(N=198)\end{array}$ & $p$-value \\
\hline Fever & $77(100)$ & $71(100)$ & $50(100)$ & $198(100)$ & - \\
\hline Myalgia & $48(62.3)$ & $50(70.4)$ & $38(76)$ & $62(31.3)$ & 0.248 \\
\hline Headache & $50(64.9)$ & $38(53.5)$ & $19(38)$ & $107(54.0)$ & 0.012 \\
\hline Conjunctival injection & $2(2.6)$ & $2(2.8)$ & $1(2)$ & $5(2.5)$ & $>0.99$ \\
\hline Cough & $12(15.8)$ & $35(49.3)$ & $15(30.6)$ & $62(31.6)$ & $<0.001$ \\
\hline Rash & $7(9.3)$ & $8(11.4)$ & $4(8)$ & $19(9.7)$ & 0.813 \\
\hline Eschar & $7(9.3)$ & $9(12.9)$ & $6(12.2)$ & $22(11.3)$ & 0.779 \\
\hline Lymphadenopathy & $13(16.9)$ & $9(12.7)$ & $3(6)$ & $25(12.6)$ & 0.196 \\
\hline Abdominal pain & $17(22.1)$ & $21(29.6)$ & $16(32.7)$ & $54(27.4)$ & 0.378 \\
\hline Nausea & $21(27.6)$ & $19(26.8)$ & $18(36)$ & $58(29.4)$ & 0.496 \\
\hline Diarrhea & $3(3.9)$ & $7(10.0)$ & $7(14.3)$ & $17(8.3)$ & 0.113 \\
\hline Jaundice & $4(5.3)$ & $10(14.5)$ & $13(26.5)$ & $27(14.0)$ & 0.004 \\
\hline Hepatomegaly & $9(11.7)$ & $9(12.9)$ & $3(6.1)$ & $21(10.7)$ & 0.474 \\
\hline Splenomegaly & $12(15.8)$ & $7(9.9)$ & $2(4.1)$ & $21(10.7)$ & 0.113 \\
\hline Seizure & $1(1.3)$ & $2(2.8)$ & $3(6)$ & $6(3.1)$ & 0.336 \\
\hline Neck stiffness & $2(2.6)$ & $1(1.4)$ & $1(2)$ & $4(2.0)$ & $>0.99$ \\
\hline Wheezing & $1(1.3)$ & $4(5.6)$ & $7(14)$ & $12(6.1)$ & 0.010 \\
\hline Crepitation & $1(1.3)$ & $21(29.6)$ & $32(65.3)$ & $54(27.5)$ & $<0.001$ \\
\hline Dyspnea & $2(2.6)$ & $15(21.1)$ & $31(64.6)$ & $48(24.6)$ & $<0.001$ \\
\hline Systolic blood pressure (mm Hg) & $117.34 \pm 13.66$ & $115.52 \pm 16.45$ & $107.88 \pm 19.68$ & $198(100)$ & 0.0058 \\
\hline Diastolic blood pressure $(\mathrm{mm} \mathrm{Hg})$ & $75.45 \pm 8.69$ & $74.70 \pm 10.06$ & $67.72 \pm 11.55$ & $198(100)$ & 0.0001 \\
\hline Pulse (beats/min) & $88.03 \pm 10.73$ & $90.82 \pm 13.61$ & $99.24 \pm 20.76$ & $198(100)$ & 0.0002 \\
\hline
\end{tabular}

Table 4: Laboratory parameters of the study cohort by severity

\begin{tabular}{|c|c|c|c|c|}
\hline Laboratory parameters & $\begin{array}{l}\text { Nonsevere }(C R S A \leq 5) \\
(N=77) \text { mean }( \pm S D) \\
\text { or median }(I Q R)\end{array}$ & $\begin{array}{l}\text { Severe }(C R S A=6-9) \\
(N=71) \text { mean }( \pm S D) \\
\text { or median }(I Q R)\end{array}$ & $\begin{array}{l}\text { Fatal }(C R S A \geq 10) \\
(N=50) \text { mean }( \pm S D) \\
\text { or median }(I Q R)\end{array}$ & $p$-value \\
\hline Hemoglobin (g/dL) & $13.01 \pm 1.55$ & $11.46 \pm 1.75$ & $11.66 \pm 1.62$ & $<0.0001$ \\
\hline Hematocrit (\%) & $37.85 \pm 4.39$ & $33.28 \pm 4.88$ & $33.96 \pm 4.65$ & $<0.0001$ \\
\hline Platelet count (×1000 cu/mm) & $171.61 \pm 82.81$ & $123.63 \pm 66.66$ & $90.38 \pm 58.15$ & $<0.0001$ \\
\hline Total leukocyte count $(\times 1000 \mathrm{cu} / \mathrm{mm})$ & $8.82 \pm 3.48$ & $12.85 \pm 19.39$ & $11.14 \pm 6.67$ & 0.138 \\
\hline Lymphocyte (\%) & $30.59 \pm 14.35$ & $25.08 \pm 12.63$ & $16.86 \pm 12.88$ & $<0.0001$ \\
\hline Monocyte (\%) & $8.47 \pm 4.91$ & $7.74 \pm 9.50$ & $5.48 \pm 3.69$ & 0.05 \\
\hline Urea (mg/dL) & $22.32 \pm 10.33$ & $31.27 \pm 17.28$ & $70.62 \pm 44.11$ & $<0.0001$ \\
\hline Creatinine (mg/dL) & $0.87 \pm 0.22$ & $0.92 \pm 0.46$ & $1.91 \pm 1.44$ & $<0.0001$ \\
\hline Sodium (mmoL/L) & $133.47 \pm 5.93$ & $132.19 \pm 4.83$ & $133.54 \pm 6.91$ & 0.327 \\
\hline Potassium (mmoL/L) & $4.12 \pm 0.54$ & $4.16 \pm 0.76$ & $4.30 \pm 0.83$ & 0.355 \\
\hline Albumin (g/dL) & $3.96 \pm 0.48$ & $2.81 \pm 0.44$ & $2.52 \pm 0.37$ & $<0.0001$ \\
\hline Globulin (g/dL) & $3.15 \pm 0.52$ & $3.31 \pm 0.54$ & $2.99 \pm 0.68$ & 0.012 \\
\hline Total bilirubin (mg/dL) & $0.66(0.49,0.95)$ & $1.30(0.57,3.55)$ & $2.55(0.90,6.09)$ & 0.0001 \\
\hline Direct bilirubin (mg/dL) & $0.26(0.17,0.0 .49)$ & $0.74(0.23,2.99)$ & $2.16(0.55,4.97)$ & 0.0001 \\
\hline Alanine transaminase (IU/L) & $67.5(44,122)$ & $84(50,127)$ & $83.5(59,114)$ & 0.186 \\
\hline Aspartate transaminase (IU/L) & $67(35.5,107)$ & $90(69,143)$ & $140(97,186)$ & 0.0001 \\
\hline Alkaline phosphatase (IU/L) & $126.5(79.5,184.5)$ & $216.5(140,318)$ & $224.5(136,355)$ & 0.0001 \\
\hline
\end{tabular}

In our study, when the cutoff for severe scrub typhus was taken as a CRSA score of $\geq 10$, a sensitivity of $51.72 \%$ and specificity of $95.90 \%$ was obtained. About $76.26 \%$ (151 of 198) of patients were correctly classified as severe, with an underestimation of $21.21 \%$ (42 patients) and an overestimation of $2.5 \%$ (5 patients). Positive predictive value was found to be $90 \%$ and negative predictive value was $71.6 \%$. 
Validation of a Clinical Risk-scoring Algorithm for Scrub Typhus Severity

Table 5: Systemic complications of the study cohort by CRSA score

\begin{tabular}{lllllr}
\hline Complications & $\begin{array}{l}(C R S A \leq 5) \\
(N=77) n(\%)\end{array}$ & $\begin{array}{l}(C R S A=6-9) \\
(N=71) n(\%)\end{array}$ & $\begin{array}{l}(C R S A \geq 10) \\
(N=50) n(\%)\end{array}$ & $\begin{array}{l}\text { Total } \\
(N=198) n(\%)\end{array}$ & $p$ value \\
\hline Cardiovascular & $3(3.9)$ & $8(11.3)$ & $18(36)$ & $29(14.6)$ & $<0.001$ \\
Respiratory & $2(2.6)$ & $6(8.4)$ & $20(40)$ & $28(14.1)$ & $<0.001$ \\
Nervous system & $2(2.6)$ & $5(7.0)$ & $10(20)$ & $17(8.6)$ & 0.002 \\
Hematology & $0(0)$ & $3(4.2)$ & $5(10)$ & $8(4.0)$ & 0.009 \\
Urinary tract & $0(0)$ & $1(1.4)$ & $22(44)$ & $23(11.6)$ & $<0.001$ \\
Gastrointestinal & $12(15.6)$ & $15(21.1)$ & $14(28)$ & $41(20.7)$ & 0.240 \\
\hline
\end{tabular}

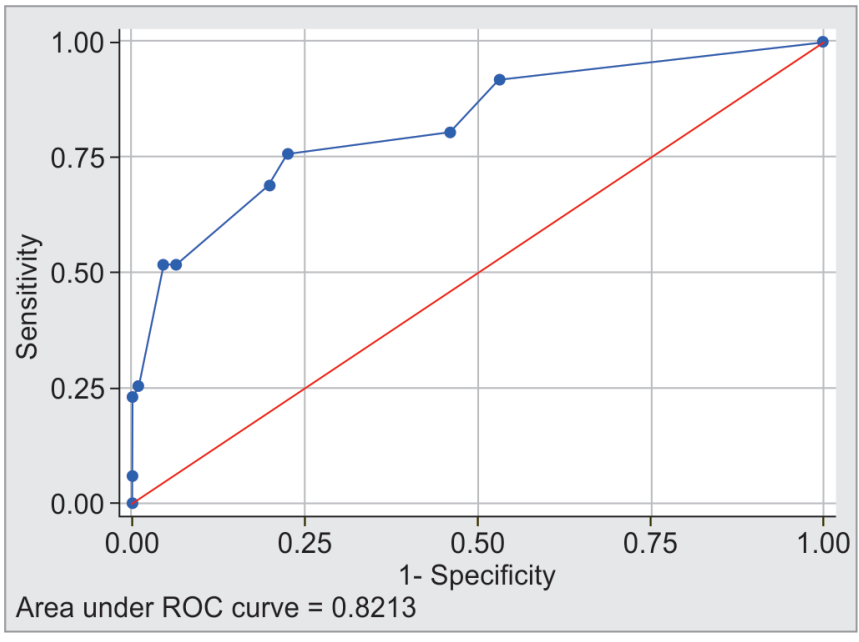

Fig. 1: Receiver-operating characteristic curve

CRSA scores of $\geq 6$ or $\geq 10$ were suboptimal for predicting the severity of scrub typhus patients in the Indian setting. In search for an optimal cutoff that provides reasonable sensitivity and specificity, we found a severity score $\geq 7$, which provides an optimal combination of sensitivity and specificity. According to the ROC curve, at a cutoff score for severe scrub typhus $\geq 7$, a sensitivity of $75.9 \%$ and specificity of $77.5 \%$ are achieved. It correctly predicted $76.77 \%$ (152 of 198) of patients as severe, with an underestimation of $10.61 \%$ (21 patients) and an overestimation of $12.63 \%$ ( 25 patients). Positive predictive value was found to be $72.5 \%$ and negative predictive value was $80.4 \%$.

The present CRSA is simple to use. It has basic and easily evaluable parameters such as age, pulse rate, crepitations, and laboratory parameters such as aspartate transaminase, serum albumin, and creatinine. The clinician can calculate these parameters at bedside easily even at the district hospital level. Early identification of these individuals with CRSA $>7$ will help in the prompt and appropriate treatment being initiated and preventing severe complications like ARDS, septic shock, and multisystem organ failure which can eventually lead to death. Such individuals could be administered intravenous antibiotics such as doxycycline and azithromycin. These flagged patient's fluid statuses should be monitored meticulously, especially in those patients with elevated creatinine and/or crepitation. They can be closely monitored for oxygenation using pulse oximetry, arterial blood gas analysis, and can be prioritized for intensive care unit management and escalation of care such as high flow nasal oxygenation, noninvasive or invasive mechanical ventilation. This risk-scoring algorithm includes the patient's symptoms, examination findings, and routinely available laboratory investigations. This will prove to be a big advantage in a country like India where most of the endemic areas of scrub are located in rural areas where health resources are limited. In this background, CRSA $\geq 7$ could be used to refer a patient to higher centers in a timely manner.

\section{Limitations}

All the patients included in this study were diagnosed based on a combination of two tests, IgM ELISA and immunofluorescence assay for scrub typhus, a chance of misclassification by the reference standard cannot be ruled out. Comparison of CRSA with other existing scoring criteria's such as quick SOFA and modified early warning score was beyond the scope of the present study. Future studies can undertake such comparisons of scoring systems for scrub typhus with the general risk scoring systems. With the CRSA score $\geq 7$, the present study showed sensitivity of $75.9 \%$ and specificity of $77.5 \%$ for predicting severity. Future studies should attempt to develop a unique scoring system for Indian scrub typhus patients, with a well-characterized scrub typhus cohort with better predictive value.

\section{Conclusion}

The present study provides additional evidence on the validity of the CRSA in a setting outside Thailand. CRSA score of $\geq 6$ to differentiate severe from nonsevere scrub typhus was suboptimal in the Indian setting with sensitivity and specificity of 80.5 and $54.1 \%$, respectively. In an Indian scrub typhus population, CRSA score of $\geq 7$ provided an optimum cutoff with sensitivity of $75.9 \%$ and specificity of $77.5 \%$. The CRSA could help clinicians in early prognostication and initiation of appropriate treatment to prevent severe complications and potentially improve the treatment outcomes in scrub typhus patients.

\section{HighLights}

- CRSA was evaluated in the Indian scrub typhus population.

- A CRSA score of $\geq 7$ provided an optimum cutoff with a sensitivity of $75.9 \%$ and specificity of $77.5 \%$.

- This CRSA can aid clinicians in early prognostication and initiation of appropriate treatment to prevent severe complications and improve outcomes.

\section{ORCID}

Shivali Gulati i https://orcid.org/0000-0002-8429-8697

Kiran Chunduru (1) https://orcid.org/0000-0001-5823-7252

Mridula Madiyal @ https://orcid.org/0000-0002-0424-9394

Maninder S Setia @ https://orcid.org/0000-0003-1291-9033

Kavitha Saravu @ https://orcid.org/0000-0001-6399-1129 


\section{References}

1. Rahi M, Gupte MD, Bhargava A, Varghese GM, Arora R. DHR-ICMR guidelines for diagnosis \& management of Rickettsial diseases in India. Indian J Med Res 2015;141(4):417-422. DOI: 10.4103/0971-5916.159279.

2. Luce-Fedrow A, Lehman ML, Kelly DJ, Mullins K, Maina AN, Stewart $\mathrm{RL}$, et al. A review of scrub typhus (Orientia tsutsugamushi and related organisms): then, now, and tomorrow. Trop Med Infect Dis 2018;3(1):8. DOI: 10.3390/tropicalmed3010008.

3. Bonell A, Lubell Y, Newton PN, Crump JA, Paris DH. Estimating the burden of scrub typhus: a systematic review. PLoS Negl Trop Dis 2017;11(9):e0005838-e0005838. DOI: 10.1371/journal. pntd.0005838.

4. Varghese GM, Janardhanan J, Trowbridge P, Peter JV, Prakash JAJ, Sathyendra S, et al. Scrub typhus in South India: clinical and laboratory manifestations, genetic variability, and outcome. Int J Infect Dis 2013;17(11):e981-e987. DOI: 10.1016/j.ijid.2013.05.017.

5. Varghese GM, Trowbridge P, Janardhanan J, Thomas K, Peter JV, Mathews $\mathrm{P}$, et al. Clinical profile and improving mortality trend of scrub typhus in South India. Int J Infect Dis 2014;23:39-43. DOI: 10.1016/j.ijid.2014.02.009.

6. Kundavaram A, Jonathan A, Nathaniel S, Varghese G. Eschar in scrub typhus: a valuable clue to the diagnosis. J Postgrad Med 2013;59(3):177-178. DOI: 10.4103/0022-3859.118033.

7. Koralur M, Bairy I, Singh R, Varma D, Stenos J. Molecular confirmation of scrub typhus infection and characterization of Orientia tsutsugamushi genotype from Karnataka, India, vol. 53. 2016. 185 p.

8. Prakash JAJ. Scrub typhus: risks, diagnostic issues, and management challenges. Res Rep Trop Med 2017;8:73-83. DOI: 10.2147/RRTM. S105602.

9. Chakraborty S, Sarma N. Scrub typhus: an emerging threat. Indian J Dermatol 2017;62(5):478-485. DOI: 10.4103/ijd.IJD_388_17.

10. Chauhan V, Thakur A, Thakur S. Eschar is associated with poor prognosis in scrub typhus. Indian J Med Res 2017;145(5):693-696. DOI: 10.4103/ijmr_1888_15.

11. Rajapakse S, Rodrigo C, Fernando D. Scrub typhus: pathophysiology, clinical manifestations and prognosis. Asian Pac J Trop Med 2012;5(4):261-264. DOI: 10.1016/S1995-7645(12)60036-4.

12. Takhar R, Bunkar M, Arya S, Mirdha N, Mohd A. Scrub typhus: a prospective, observational study during an outbreak in Rajasthan, India. Natl Med J India 2017;30(2):69-72.

13. Attur RP, Kuppasamy S, Bairy M, Nagaraju SP, Pammidi NR, Kamath $V$, et al. Acute kidney injury in scrub typhus. Clin Exp Nephrol 2013;17(5):725-729. DOI: 10.1007/s10157-012-0753-9.
14. Jayaprakash V, Vamsikrishna M, Indhumathi E, Jayakumar M. Scrub typhus-associated acute kidney injury: a study from a South Indian Tertiary Care Hospital. Saudi J Kidney Dis Transpl 2019;30(4):883. DOI: 10.4103/1319-2442.265464.

15. Viswanathan S, Muthu V, Iqbal N, Remalayam B, George T. Scrub typhus meningitis in South India - a retrospective study. PLoS One 2013;8(6):e66595. DOI: 10.1371/journal.pone.0066595.

16. Taylor AJ, Paris DH, Newton PN. A systematic review of mortality from untreated scrub typhus (Orientia tsutsugamushi). PLoS Negl Trop Dis 2015;9(8):e0003971. DOI: 10.1371/journal.pntd.0003971.

17. Jain D, Nand N, Giri K, Bhutani J. Scrub typhus infection, not a benign disease: an experience from a tertiary care center in Northern India. Med Pharm Rep 2019;92(1):36-42. DOI: 10.15386/cjmed-1088.

18. Shivalli S. Diagnostic evaluation of rapid tests for scrub typhus in the Indian population is needed. Infect Dis Poverty 2016;5(1):40. DOI: 10.1186/s40249-016-0137-6.

19. España PP, Capelastegui A, Gorordo I, Esteban C, Oribe M, Ortega $M$, et al. Development and validation of a clinical prediction rule for severe community-acquired pneumonia. Am J Respir Crit Care Med 2006;174(11):1249-1256. DOI: 10.1164/rccm.200602-1770C.

20. Tanner L, Schreiber M, Low JGH, Ong A, Tolfvenstam T, Lai YL, et al. Decision tree algorithms predict the diagnosis and outcome of dengue fever in the early phase of illness. PLOS Negl Trop Dis 2008;2(3):e196. DOI: 10.1371/journal.pntd.0000196.

21. Lee VJ, Lye DC, Sun Y, Leo YS. Decision tree algorithm in deciding hospitalization for adult patients with dengue haemorrhagic fever in Singapore. Trop Med Int Health 2009;14(9):1154-1159. DOI: 10.1111/j.1365-3156.2009.02337.x.

22. Butt E, Foster JA, Keedwell E, Bell JE, Titball RW, Bhangu A, et al. Derivation and validation of a simple, accurate and robust prediction rule for risk of mortality in patients with Clostridium difficile infection. BMC Infect Dis 2013;13(1):316. DOI: 10.1186/1471-2334-13-316.

23. Mohapatra M, Das P. The malaria severity score: a method for severity assessment and risk prediction of hospital mortality for falciparum malaria in adults. J Assoc Physicians India 2009;57:119-126.

24. Sriwongpan $P$, Krittigamas $P$, Tantipong H, Patumanond J, Tawichasri C, Namwongprom S. Clinical risk-scoring algorithm to forecast scrub typhus severity. Risk Manag Healthc Policy 2013;7:11-17. DOI: 10.2147/ RMHP.S55305.

25. Sriwongpan $\mathrm{P}$, Patumanond J, Krittigamas $\mathrm{P}$, Tantipong H, Tawichasri C, Namwongprom S. Validation of a clinical risk-scoring algorithm for severe scrub typhus. Risk Manag Healthc Policy 2014;7:29-34. DOI: 10.2147/RMHP.S56974. 